\title{
Nodal free geometric phases: Concept and application to geometric quantum computation
}

\author{
Marie Ericsson ${ }^{\text {a }}$, David Kult ${ }^{\mathrm{b}}$, Erik Sjöqvist ${ }^{\mathrm{b}, *}$, Johan Åberg ${ }^{\mathrm{a}}$ \\ ${ }^{a}$ Centre for Quantum Computation, Department of Applied Mathematics and \\ Theoretical Physics, University of Cambridge, Wilberforce Road, Cambridge CB3 \\ oWA, United Kingdom \\ ${ }^{\mathrm{b}}$ Department of Quantum Chemistry, Uppsala University, Box 518, Se-751 20 \\ Uppsala, Sweden
}

\begin{abstract}
Nodal free geometric phases are the eigenvalues of the final member of a parallel transporting family of unitary operators. These phases are gauge invariant, always well-defined, and can be measured interferometrically. Nodal free geometric phases can be used to construct various types of quantum phase gates.
\end{abstract}

Key words: Geometric phase; Quantum gates; Interferometry PACS: 03.65.Vf, 03.67.Lx, 07.60.Ly

Geometric phases in noncyclic evolution [1] are undefined if the initial and final states are orthogonal. Similarly, off-diagonal geometric phases [2] are undefined for cyclic evolution. Thus, it appears to be a general fact that geometric phases have nodal points at which they are undefined. Nevertheless, these phases probe the geometry of the ray space, which is free from any singularities. This observation raises the question if the concept of geometric phase can be modified so that it reflects the absence of singularities in ray space. Here, we propose such a modified concept, which we shall call nodal free geometric phases.

Recently, geometric phases have been suggested to play a role in the design of quantum gates that may be robust to certain kinds of errors. This idea was

\footnotetext{
* Corresponding author.

Email addresses: M.Ericsson@damtp.cam.ac.uk (Marie Ericsson), david.kult@kvac.uu.se (David Kult), eriks@kvac.uu.se (Erik Sjöqvist), J.Aberg@damtp.cam.ac.uk (Johan Åberg).
} 
first put forward in the case of cyclic adiabatic evolution [3], and was subsequently implemented in the nonadiabatic [4] and noncyclic [5,6] contexts. The desired geometric properties may also occur when the dynamical phase becomes proportional to the geometric phase, leading to the notion of unconventional geometric quantum computation [7. Here, we propose the nodal free geometric phases as another conceptual basis for the construction of quantum gates.

Let $U(s), s \in[0,1]$, be an arbitrary one-parameter family of unitary operators acting on an $N$-dimensional Hilbert space $\mathcal{H}$. We require that $U(0)=\hat{1}$, i.e., the identity operator on $\mathcal{H}$. This family is said to be parallel transporting if there exists an orthonormal basis $\psi=\left\{\left|\psi_{k}\right\rangle\right\}_{k}$ of $\mathcal{H}$ such that each $U(s)\left|\psi_{k}\right\rangle$ satisfies the standard parallel transport condition [8,9,10]

$$
\left\langle\psi_{k}\left|U^{\dagger}(s) \dot{U}(s)\right| \psi_{k}\right\rangle=0
$$

Not all $U(s)$ has this property, e.g., for a qubit $(N=2), e^{-i s \sigma_{z}}$ is parallel transporting (any pair of orthogonal vectors that represent states on the equator of the Bloch sphere will do), while $e^{-i s \sigma_{x}} e^{-i s \sigma_{z}}$ is not.

Now, let $U_{\psi}^{\|}(s), s \in[0,1]$ and $U_{\psi}^{\|}(0)=\hat{1}$, be a family of unitary operators that parallel transport the orthonormal basis $\psi=\left\{\left|\psi_{k}\right\rangle\right\}_{k}$. We are interested in the properties of $U_{\psi}^{\|}(1)$. Consider the $N \times N$ matrix

$$
\boldsymbol{U}_{\psi}^{\|}=\left(\begin{array}{ccc}
\sigma_{11} & \ldots & \sigma_{1 N} \\
\vdots & \ddots & \vdots \\
\sigma_{N 1} & \ldots & \sigma_{N N}
\end{array}\right)
$$

where $\sigma_{k l}=\left\langle\psi_{k}\left|U_{\psi}^{\|}(1)\right| \psi_{l}\right\rangle$, i.e., $\boldsymbol{U}_{\psi}^{\|}$is the matrix representation of $U_{\psi}^{\|}(1)$ with respect to $\psi$. Since $\boldsymbol{U}_{\psi}^{\|}$is a unitary matrix, it has $N$ unit modulus eigenvalues. These eigenvalues coincide with the standard geometric phase factors in cyclic evolution [11,12,13] if $\boldsymbol{U}_{\psi}^{\|}$is diagonal, i.e., when all $U_{\psi}^{\|}(s)\left|\psi_{k}\right\rangle$ undergo cyclic evolution. In the noncyclic case, $\boldsymbol{U}_{\psi}^{\|}$has nonzero off-diagonal elements. The corresponding eigenvalues are still gauge invariant phase factors, i.e., invariant under transformations of the form $\left|\psi_{k}\right\rangle \rightarrow e^{i \alpha_{k}}\left|\psi_{k}\right\rangle$, but differ from the standard geometric phase factors in noncyclic evolution [1]. In particular, the eigenvalues are always well-defined, i.e., there are no nodal points where they become undefined. These eigenvalues are the nodal free geometric phase factors of the set of paths $\left\{\Pi\left[U_{\psi}^{\|}(s)\left|\psi_{k}\right\rangle\right]\right\}_{k}$ in ray space, $\Pi$ being the projection map [11].

To prove the gauge invariance of the nodal free geometric phase factors, we 
note that $\sigma_{k l} \rightarrow \sigma_{k l} e^{-i\left(\alpha_{k}-\alpha_{l}\right)}$, under $\left|\psi_{k}\right\rangle \rightarrow e^{i \alpha_{k}}\left|\psi_{k}\right\rangle$. Thus, $\boldsymbol{U}_{\psi}^{\|} \rightarrow \boldsymbol{V} \boldsymbol{U}_{\psi}^{\|} \boldsymbol{V}^{\dagger}$, where $\boldsymbol{V}=\operatorname{diag}\left[e^{-i \alpha_{1}}, e^{-i \alpha_{2}}, \ldots, e^{-i \alpha_{N}}\right]$, from which it follows that the eigenvalues are preserved.

Similarly as for the standard [1] and off-diagonal [2] geometric phase factors the nodal free geometric phase factors can be defined in terms of gauge-invariant quantities. The eigenvalues $\lambda$ of $\boldsymbol{U}_{\psi}^{\|}$are solutions of the secular equation $\operatorname{det}\left(\boldsymbol{U}_{\psi}^{\|}-\lambda \mathbf{1}\right)=0, \mathbf{1}$ being the $N \times N$ unit matrix. Expanding the determinant yields an equation that involves $\gamma_{j}^{(1)}=\sigma_{j j}$ and $\gamma_{j_{1} \ldots j_{l}}^{(l)} \equiv \sigma_{j_{1} j_{l}} \cdots \sigma_{j_{2} j_{1}}$, $l=2,3 \ldots, N$, which are the gauge invariant quantities that define the standard and off-diagonal geometric phase factors $\Phi\left[\gamma^{(l)}\right]$, where $\Phi[z]=z /|z|$ for any nonzero complex number $z$. For instance, for $N=3$ we have

$$
\begin{aligned}
& -\lambda^{3}+\left(\gamma_{1}^{(1)}+\gamma_{2}^{(1)}+\gamma_{3}^{(1)}\right) \lambda^{2} \\
& -\left(\gamma_{1}^{(1)} \gamma_{2}^{(1)}+\gamma_{2}^{(1)} \gamma_{3}^{(1)}+\gamma_{3}^{(1)} \gamma_{1}^{(1)}-\gamma_{12}^{(2)}-\gamma_{23}^{(2)}-\gamma_{31}^{(2)}\right) \lambda \\
& +\gamma_{123}^{(3)}+\gamma_{132}^{(3)}-\gamma_{12}^{(2)} \gamma_{3}^{(1)}-\gamma_{23}^{(2)} \gamma_{1}^{(1)}-\gamma_{31}^{(2)} \gamma_{2}^{(1)} \\
& +\gamma_{1}^{(1)} \gamma_{2}^{(1)} \gamma_{3}^{(1)}=0 .
\end{aligned}
$$

The solutions of this equation are fully determined by all the $\gamma$ 's.

One may derive some results for the off-diagonal geometric phases using the above nodal free scenario. We first note that a central motivation for Manini and Pistolesi [2] to introduce the concept of off-diagonal geometric phases was to retain geometric phase information of the evolution in cases where the standard geometric phase factors were undefined. In the present context, we can indeed see that this must always be the case since if, for a given Hilbert space dimension $N$, all $\gamma^{\prime}$ 's happened to vanish and thereby all $\Phi[\gamma]$ were undefined, the secular equation would reduce to $\lambda^{N}=0$, which contradicts the fact that $|\lambda|=1$ (see also Ref. [14]). Furthermore, one may see that the $\lambda^{0}$ coefficient of the eigenvalue equation has the structure $\gamma^{(N)}+\gamma^{(N-1)} \gamma^{(1)}+$ $\gamma^{(N-2)}\left[\gamma^{(1)}\right]^{2}+\gamma^{(N-2)} \gamma^{(2)}+\ldots+\left[\gamma^{(1)}\right]^{N}$. This term must be nonzero, since if it would vanish, then $\lambda=0$ would be a solution of the eigenvalue equation. It follows, in particular, that $\gamma^{(l \neq n)}=0$ for some fixed $n$ is only possible for the 'extremal' $n=1$ and $n=N$.

Next, we consider the relation between the nodal free phase factors and the cyclic geometric phase factors [11,12,13]. Let $\left|\phi_{k}\right\rangle$ be orthonormal eigenvectors of $U_{\psi}^{\|}(1)$. These are cyclic states, i.e., $U_{\psi}^{\|}(1)\left|\phi_{k}\right\rangle=\lambda_{k}\left|\phi_{k}\right\rangle$, where $\lambda_{k}$ are the nodal free geometric phase factors. Note that the eigenvectors are in general not parallel transported by $U_{\psi}^{\|}(s)$. The nodal free phase factors $\lambda_{k}$ differ from the standard cyclic geometric phase factors $e^{i \beta_{k}}$ [11,12]13] associated with the paths $\Pi\left[U_{\psi}^{\|}(s)\left|\phi_{k}\right\rangle\right]$ in ray space. To see this we first note that given the family 
of unitaries $U_{\psi}^{\|}(s)$ the Schrödinger equation uniquely determines the family of Hamiltonians $H(s)=i \dot{U}_{\psi}^{\|}(s) U_{\psi}^{\|^{\dagger}}(s)$ that generates $U_{\psi}^{\|}(s)$. If we let $\tau_{k}$ be the phase associated with the nodal free phase factor, i.e., $\lambda_{k}=e^{i \tau_{k}}$, we can use the technique in Ref. [11] (see Eq. (3) in Ref. [11]) to find that the cyclic geometric phases $\beta_{k}$ of $\left|\phi_{k}\right\rangle$ are

$$
\beta_{k}=\tau_{k}+\int_{0}^{1}\left\langle\phi_{k}(s)|H(s)| \phi_{k}(s)\right\rangle d s
$$

which we can rewrite as

$$
e^{i \beta_{k}}=\lambda_{k} e^{i \int_{0}^{1}\left\langle\phi_{k}|H(s)| \phi_{k}\right\rangle d s}
$$

This establishes an explicit relation between the nodal free geometric phase factors and the geometric phase factors associated with the states that undergo cyclic evolution for the family $U_{\psi}^{\|}(s)$. As can be seen, these two differ by the dynamical phase factors of the eigenvectors $\left|\phi_{k}\right\rangle$. It is to be noted that, since the family of Hamiltonians is uniquely determined, these dynamical phase factors do not introduce any ambiguity in the definition of the nodal free geometric phases.

As a final note on the general properties of the nodal free geometric phases, we point out that these can be measured in interferometry. A beam of particles with some internal degree of freedom (e.g., spin or polarization) prepared in the internal state $|\varphi\rangle$ is splitted by a 50-50 beam-splitter into two beams. In one of the resulting beams, the internal state is transformed by the unitary operators $U_{\psi}^{\|}(s), s \in[0,1]$, that parallel transport the basis $\psi=\left\{\left|\psi_{k}\right\rangle\right\}_{k}$, and a variable $\mathrm{U}(1)$ phase shift $e^{i \chi}$ is applied to the other beam. The two beams are brought back to interfere at a second beam-splitter. The resulting interference pattern is determined by the complex-valued quantity $F(\varphi)=\left\langle\varphi\left|U_{\psi}^{\|}(1)\right| \varphi\right\rangle$ in that the intensity measured in one of the output beams reads [15]

$$
\mathcal{I} \propto 1+|F(\varphi)| \cos [\chi-\arg F(\varphi)]
$$

Explicitly,

$$
F(\varphi)=\sum_{k}\left|\left\langle\phi_{k} \mid \varphi\right\rangle\right|^{2} \lambda_{k}
$$

where $\sum_{k}\left|\left\langle\phi_{k} \mid \varphi\right\rangle\right|^{2}=1$, i.e., the interference function $F(\varphi)$ is a convex combination of the nodal free geometric phase factors. It follows that the interference oscillations are shifted by the nodal free geometric phases $\arg \lambda_{k}$ if and only if $|\varphi\rangle$ coincide with $\left|\phi_{k}\right\rangle$, in case of which $|F(\varphi)|=1$. Thus, the nodal free geometric phases can be obtained by varying the input internal state $\varphi$ until unit visibility is attained. 
To illustrate the theory with a specific example, let us analyze the nodal free geometric phases in the qubit case in some detail. Let $\eta=\left|\left\langle\psi_{1}\left|U_{\psi}^{\|}(1)\right| \psi_{1}\right\rangle\right|=$ $\left|\left\langle\psi_{2}\left|U_{\psi}^{\|}(1)\right| \psi_{2}\right\rangle\right|$ measure the degree of cyclicity and let $\Omega$ be the solid angle enclosed by the path traced out by the Bloch vector and the shortest geodesics connecting the end-points. In terms of these quantities, we have $\gamma_{1}^{(1)}=\left(\gamma_{2}^{(1)}\right)^{*}=\eta e^{-i \Omega / 2}$ and $\gamma_{12}^{(2)}=\eta^{2}-1$. Thus, if $\eta=0$ then the geometric phase factors $\Phi\left[\gamma_{1}^{(1)}\right]$ and $\Phi\left[\gamma_{2}^{(1)}\right]$ are undefined, but the off-diagonal phase factor $\Phi\left[\gamma_{12}^{(2)}\right]=-1$. If $\eta=1$ then the off-diagonal geometric phase factor $\Phi\left[\gamma_{12}^{(2)}\right]$ is undefined and $\Phi\left[\gamma_{1}^{(1)}\right]=\left(\Phi\left[\gamma_{2}^{(1)}\right]\right)^{*}=e^{-i \Omega / 2}$. On the other hand, the nodal free geometric phase factors, i.e., the eigenvalues of $\boldsymbol{U}_{\psi}^{\|}$, are always defined. The secular equation reads $\lambda^{2}-\left(\gamma_{1}^{(1)}+\gamma_{2}^{(2)}\right) \lambda+\gamma_{1}^{(1)} \gamma_{2}^{(1)}-\gamma_{12}^{(2)}=0$, which gives the eigenvalues

$$
\lambda_{ \pm}=\eta \cos (\Omega / 2) \pm i \sqrt{1-\eta^{2} \cos ^{2}(\Omega / 2)}
$$

with corresponding eigenstates $\left|\phi_{ \pm}\right\rangle$. We note that $1 \geq \eta^{2} \cos ^{2}(\Omega / 2)$ and $\left|\lambda_{ \pm}\right|=$ 1. For 'bit flip' evolution $(\eta=0)$, the geodesically closed solid angle $\Omega$ is undefined since there is an infinite number of ways to close the path by a geodesics, and all these closures yield different solid angles. However, whatever geodesic closure we choose, the nodal free geometric phase factors are given by $\pm i$. For cyclic evolution $(\eta=1)$, we obtain the two eigenvalues $\lambda_{ \pm}=$ $\cos (\Omega / 2) \pm i|\sin (\Omega / 2)|$. Hence, as expected we find the two cyclic geometric phase factors $e^{i \Omega / 2}$ and $e^{-i \Omega / 2}$. More precisely, $\lambda_{+,-}=\Phi\left[\gamma_{2,1}^{(1)}\right]$ for $\sin (\Omega / 2)>0$ and $\lambda_{+,-}=\Phi\left[\gamma_{1,2}^{(1)}\right]$ for $\sin (\Omega / 2)<0$, i.e., the labeling of the eigenstates depends on the sign of $\sin (\Omega / 2)$ (note that $\lambda_{+}=\lambda_{-}$when $\sin (\Omega / 2)=0$, which allows for the 'flip' of the labeling).

The cyclic geometric phases arising from parallel transport have been considered for implementations of phase gates [16]. In such applications, the parallel transported and computational bases coincide. The nodal free geometric phases provide an alternative implementation of phase gates, if we let the computational basis coincide with the eigenstates of $U_{\psi}^{\|}(1)$. Here, the computational basis in general does not coincide with the parallel transported basis. In the single qubit case, considered above, we choose the computational basis $|0\rangle \equiv\left|\phi_{-}\right\rangle$and $|1\rangle \equiv\left|\phi_{+}\right\rangle$. The nodal free geometric phases may thus be interpreted as a realization of the one-qubit phase gate $U=\lambda_{-}|0\rangle\left\langle 0\left|+\lambda_{+}\right| 1\right\rangle\langle 1|$. Such a phase gate is fully determined by the quantities $\gamma_{1}^{(1)}, \gamma_{2}^{(1)}$, and $\gamma_{12}^{(2)}$ via the eigenvalue equation. In the special case of cyclic evolution of the parallel transported basis, $U$ reduces to the standard nonadiabatic geometric phase gate $\gamma_{1}^{(1)}|0\rangle\left\langle 0\left|+\gamma_{2}^{(1)}\right| 1\right\rangle\left\langle 1\left|=e^{-i \Omega / 2}\right| 0\right\rangle\left\langle 0\left|+e^{i \Omega / 2}\right| 1\right\rangle\langle 1|$, which is sensitive to changes in the solid angle $\Omega$. On the other hand, the off-diagonal geometric phase factor $\Phi\left[\gamma_{12}^{(2)}\right]$ is either undefined (for cyclic evolution $\eta=1$ ) or -1 (for $0 \leq \eta<1$ ). This $\Omega$ independence can be understood geometrically by interpreting the second order off-diagonal geometric phase in terms of a loop 

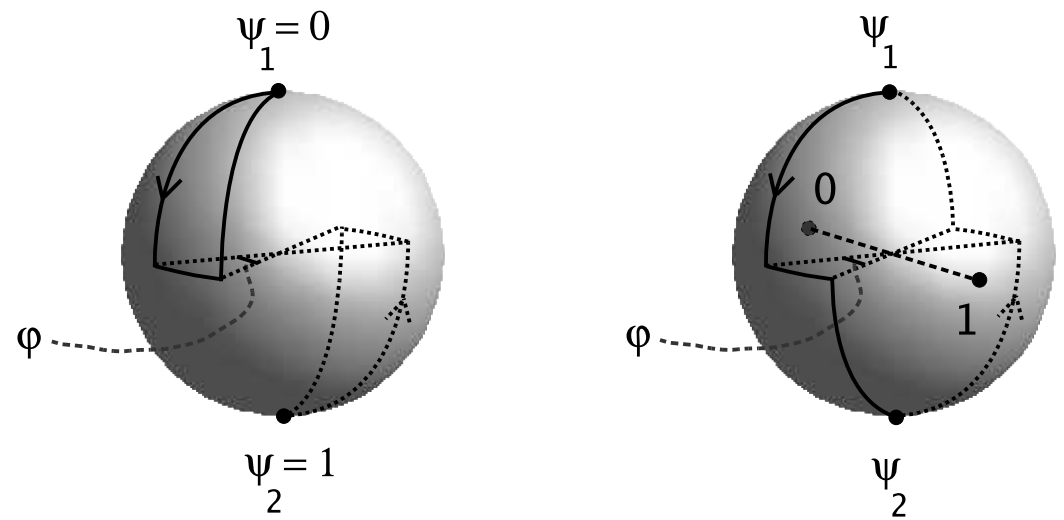

Fig. 1. Paths on Bloch sphere for phase shift gate (left panel) and phase flip gate (right panel), based on the standard and nodal free geometric phases, respectively. These paths can be realized for instance by applying magnetic fields sequentially in three different directions to a spin $-\frac{1}{2}$ particle. The phase shift gate is determined by the solid angle $\Omega=\varphi$. Here, the computational and parallel transported bases coincide. The phase flip gate depends only on the off-diagonal geometric phase $\arg \gamma_{12}^{(2)}$. This phase equals half the solid angle enclosed by the loop sequentially traced out by the path starting at $\left|\psi_{1}\right\rangle$, followed by the path starting at $\left|\psi_{2}\right\rangle$, i.e., $\arg \gamma_{12}^{(2)}=\pi$ [2]. Thus, the nodal free geometric phase factors $\pm \sqrt{\gamma_{12}^{(2)}}= \pm i$ that define this gate are $\varphi$ independent. Note, though, that the precise location of the computational basis indicated by ' 0 ' and ' 1 ', on the equator of the Bloch sphere, depends on $\varphi$. One can show that $|0\rangle=\frac{1}{\sqrt{2}}\left(\left|\psi_{1}\right\rangle+e^{-i(\varphi-\pi) / 2}\left|\psi_{2}\right\rangle\right)$ and $|1\rangle=\frac{1}{\sqrt{2}}\left(\left|\psi_{1}\right\rangle+e^{-i(\varphi+\pi) / 2}\left|\psi_{2}\right\rangle\right)$ for this phase flip gate.

that always encloses the solid angle $2 \pi$. This property has been demonstrated experimentally for neutron spin [17] and suggests that the off-diagonal geometric phases may be a useful component for the design of quantum gates. Since $\gamma_{12}^{(2)}=\gamma_{21}^{(2)}$, it seems at first sight that only a trivial phase gate can be implemented based on the off-diagonal geometric phase. However, in the nodal free geometric phase scenario with $\eta=0$, we obtain

$$
i Z=\sqrt{\gamma_{12}^{(2)}}|0\rangle\left\langle 0\left|-\sqrt{\gamma_{12}^{(2)}}\right| 1\right\rangle\langle 1|=i| 0\rangle\langle 0|-i| 1\rangle\langle 1|,
$$

which, up to the unimportant overall phase factor $i$, is a nontrivial phase flip gate. Thus, the nodal free geometric phase concept makes it possible to use offdiagonal geometric phases in the design of quantum gates. The robustness with respect to changes in $\Omega$ suggests that this gate implementation would have an inherent stability against noise in this parameter. Realizations of paths on the Bloch sphere leading to the phase shift and phase flip gates using the standard and nodal free geometric phases, respectively, are shown in Fig. (11). 
To develop this idea further, we consider two-qubit geometric phase gates, in case of which the relevant Hilbert space is four-dimensional. One may seek for a similar path independence as in the above phase flip gate by looking for implementations that only involve the fourth order $(l=4)$ off-diagonal geometric phases. To this end, we assume that $U_{\psi}^{\|}(1)$ takes the matrix form

$$
\boldsymbol{U}_{\psi}^{\|}(1)=\left(\begin{array}{cccc}
0 & \sigma_{12} & 0 & 0 \\
0 & 0 & \sigma_{23} & 0 \\
0 & 0 & 0 & \sigma_{34} \\
\sigma_{41} & 0 & 0 & 0
\end{array}\right)
$$

in the parallel transported basis. The eigenvalue equation reads $\lambda^{4}-\gamma_{1234}^{(4)}=0$, yielding the nodal free geometric phase factors $\lambda_{k}=e^{i k \pi / 2}\left(\gamma_{1234}^{(4)}\right)^{1 / 4}, k=$ $0, \ldots, 3$. The parallel transporting nature of the family $U_{\psi}^{\|}(s)$ implies that $U_{\psi}^{\|}(1) \in \mathrm{SU}(4)$, i.e., $\operatorname{det} U_{\psi}^{\|}(1)=1$. Thus, $\gamma_{1234}^{(4)}=-1$ so that $\lambda_{k}=e^{i(2 k+1) \pi / 4}$, $k=0, \ldots, 3$. Let us assume that there is a physically natural tensor product decomposition of the Hilbert space such that the eigenvectors of $U_{\psi}^{\|}(1)$ coincide with the computational product basis. For instance, if $\left|\phi_{0}\right\rangle=|00\rangle,\left|\phi_{1}\right\rangle=$ $|01\rangle,\left|\phi_{2}\right\rangle=|11\rangle,\left|\phi_{3}\right\rangle=|10\rangle$, we obtain the conditional gate

$$
B=e^{i \frac{\pi}{4}}|00\rangle\left\langle 00\left|+e^{i \frac{3 \pi}{4}}\right| 01\right\rangle\left\langle 01\left|+e^{i \frac{5 \pi}{4}}\right| 11\right\rangle\left\langle 11\left|+e^{i \frac{7 \pi}{4}}\right| 10\right\rangle\langle 10| .
$$

On the other hand, if $\left|\phi_{0}\right\rangle=|00\rangle,\left|\phi_{1}\right\rangle=|11\rangle,\left|\phi_{2}\right\rangle=|10\rangle,\left|\phi_{3}\right\rangle=|01\rangle$, we obtain the product gate

$$
Z \otimes S=(|0\rangle\langle 0|-| 1\rangle\langle 1|) \otimes\left(e^{i \pi / 4}|0\rangle\left\langle 0\left|-e^{-i \pi / 4}\right| 1\right\rangle\langle 1|\right)
$$

where $S$ is the phase or $\pi / 4$ gate. We may continue to apply this idea for more qubits. In particular, we notice that the procedure admits a realization of the $\pi / 8$ gate using three qubits, based entirely on the extremal $\gamma_{12 \ldots 8}^{(8)}$. This $\pi / 8$ gate is an important ingredient to achieve universal fault tolerant quantum computation [18].

In conclusion, we introduce a family of geometric phases for parallel transporting unitary evolutions. These are explicitly constructed from gauge invariant quantities, but nevertheless do not have the nodal structure concomitant to the noncyclic and off-diagonal geometric phases. We furthermore show that the nodal free geometric phases have an operational meaning in the sense that they can be measured using interferometry. We point out that nodal free geometric phases could be useful in geometric quantum computation as they 
may show robustness features that are not shared by the standard geometric phases.

M.E. acknowledges financial support from the Leverhulme Trust. E.S. acknowledges financial support from the Swedish Research Council. J.A. wishes to thank the Swedish Research Council for financial support and the Centre for Quantum Computation at DAMTP, Cambridge, for hospitality. The work by

M.E. and J.A. was supported by the European Union through the Integrated Project QAP (IST-3-015848), SCALA (CT-015714), SECOQC and the QIP IRC (GR/S821176/01).

\section{References}

[1] J. Samuel, R. Bhandari, Phys. Rev. Lett. 60 (1988) 2339.

[2] N. Manini, F. Pistolesi, Phys. Rev. Lett. 85 (2000) 3067.

[3] P. Zanardi, M. Rasetti, Phys. Lett. A 264 (1999) 94.

[4] W. Xiang-Bin, M. Keiji Phys. Rev. Lett. 87 (2002) 097902.

[5] A. Friedenauer, E. Sjöqvist, Phys. Rev. A 67 (2003) 024303.

[6] D. Kult, J. Åberg, E. Sjöqvist, Phys. Rev. A 74 (2006) 022106.

[7] S.-L. Zhu, Z.D. Wang, Phys. Rev. Lett. 91 (2003) 187902.

[8] J. Anandan, L. Stodolsky, Phys. Rev. D 35 (1987) 2597.

[9] J. Anandan, Phys. Lett. A 129 (1988) 201.

[10] J. Anandan, Y. Aharonov, Phys. Rev. D 38 (1988) 1863.

[11] Y. Aharonov, J. Anandan, Phys. Rev. Lett. 58 (1987) 1593.

[12] J. Anandan, Phys. Lett. A 133 (1988) 171.

[13] L.-A. Wu, Phys. Rev. A 50 (1994) 5317.

[14] D. Kult, J. Åberg, E. Sjöqvist, Europhys. Lett. 78 (2007) 60004.

[15] A.G. Wagh, V.C. Rakhecha, Phys. Lett. A 197 (1995) 107.

[16] X.-Q. Li, L.-X. Cen, G. Huang, L. Ma, Y. Yan, Phys. Rev. A 66 (2002) 042320.

[17] Y. Hasegawa, R. Loidl, M. Baron, G. Badurek, H. Rauch, Phys. Rev. Lett. 87 (2001) 070401.

[18] P.O. Boykin, T. Mor, M. Pulver, V. Roychowdhury, F. Vatan, Inform. Process. Lett. 75 (2000) 101. 